\title{
DESIGN THINKING ONLINE: APPROACHING CONSTRUCTIVE LEARNING IN FOOD SCIENCE
}

\author{
Marco Garcia-Vaquero \\ School of Agriculture and Food Science \\ University College Dublin, Belfield, Dublin 4, Ireland
}

\begin{abstract}
This work aims to re-structure to an online environment and address the needs for integration of a module focused on the Science of Food Packaging delivered face-to-face. This was performed by identifying threshold concepts and applying the principles of constructive alignment when designing new learning activities and assessments adapted to an online environment for the module "Design Thinking for Food Packaging" within the framework of the Food Science Programme. Several complex threshold concepts were identified, as well as the need to integrate the contents of the module, while maintaining curriculum coherence and aligning learning activities and assessment strategies. The re-structured learning activities online included both asynchronous and synchronous activities delivered through the online platforms Brightspace and Zoom, respectively. Synchronous online learning via Zoom was designed as a problem based learning scenario to be solved by groups of learners using the design thinking methodology as scaffolding for problem solving. Asynchronous materials supported the synchronous learning, serving as the initial stepping stones for the learning and research of each group into the threshold concepts identified for this discipline. The assessment strategy focused on continuous assessment of individual and group learning and introduced the learners to the "reflective practice" via individual online learning journals. Future work is necessary to evaluate the learners' perception of the changes implemented when adapting the module to an online environment. The re-designed module online clearly aligns previous module's learning outcomes with the new learning activities and assessment strategies, facilitating the integration of knowledge while developing the interpersonal skills of the learners feeding-forward into their future professional practice as food scientists.
\end{abstract}

\section{KEYWORDS}

Constructive Alignment, Threshold, Integration, Design Thinking, Online learning, Food Science

\section{INTRODUCTION}

The module "Design Thinking for Food Packaging" aims to integrate the knowledge of key concepts of food packaging (material science and regulatory requirements in food packaging) with previous learning in the curriculum, such as food chemistry, product development and food technology. The original module was delivered face-to-face via lectures and tutorials as well as parallel guided problem solving activities in which the learners interact with industries, acting as consultants to solve their queries. This approach has certain innovative aspects related to the contact of the learners with real industries and active learning activities, however, the contents of the module were poorly integrated. Thereby, the aforementioned consultation work with industry was posed by each company to the groups and the problems identified by the learners rarely related to food packaging which is the main focus of the theoretical content covered during the module, resulting in two differentiated "works" within the module that caused fragmented learning and thus, did not allow a deeper learning and understanding of concepts relevant for their professional development. Similar problems of fragmented learning were also appreciated in modules related to Food Science and were addressed by re-designing the learning activities and aligning them with the learning objectives (Sjöö et al., 2010).

Moreover, due to Cov-19 restrictions to face-to-face delivery of the content, there was a need to limit the content delivered face-to-face and thus, the module had to be re-designed by exploring the use of alternative online tools and technologies, addressing correctly the original learning outcomes of the face-to-face module while ensuring a challenging and active learning experience that could be effective even if the restrictions 
due to Cov-19 crisis were eased. Moreover, the integration issues of the learning activities of the face-to-face module also needed to be addressed when re-designing the module by elucidating novel and interactive online learning activities and assessments more aligned with the proposed learning outcomes while maintaining the active learning or learning by doing approach of the original module design.

This work focuses on the modifications and novel aspects of the re-designed work of the module "Design Thinking for Food Packaging" to adapt it to an online environment, while addressing the need for integration of the previous content delivered face-to-face in the module. The identification of threshold concepts and the application of the principles of constructive alignment when designing new learning activities and assessment strategies adapted to an online environment are also described, as well as the implementation plan performed to ensure the current and future delivery of the learning objectives of this module within the framework of the Food Science Programme even after the Cov-19 crisis.

\section{THRESHOLD CONCEPTS}

A threshold concept as described by Meyer and Land (2003) is an "akin to a portal, opening up a new and previously inaccessible way of thinking about something. It represents a transformed way of understanding, or interpreting, or viewing something without which the learner cannot progress". Several threshold concepts were identified in the module:

(1) Food products and raw materials. Understanding the needs of multiple food products and how these needs may influence their packaging materials. Thereby, when packaging fresh vegetables (salad leaves or full lettuce with roots) the needs of packaging both products are different (Sandhya, 2010). Thus, understanding the food products and raw materials in the context of food packaging is a threshold in this module.

(2) Food packaging materials and regulations. The physical properties of different polymers and their chemistry may influence their use depending on the intended use of the food products (Arvanitoyannis and Kotsanopoulos, 2014, Vera et al., 2020). The understanding of the basic chemistry, physical properties of the materials together with the regulations available in the country is a threshold concept involving broad knowledge of multiple disciplines relevant for their professional activities in the future.

(3) Food processing and manufacturing. The use of one packaging material over another may have strong influence on the industrial machinery and processing of the product at industrial level with implications for the cost of the product and also the time the product may be preserved (shelf-life) (Sarker, 2020). This is a threshold concept involving previous knowledge of the learners related to food technology and deep thinking on the bigger picture and industrial implications of packaging.

(4) Consumer acceptability and market. Even when the packaging materials follow the regulations and they are good for the preservation of the food product, the acceptance of the food can be drastically affected by the materials of choice to package the product and may result in decreased sells. The understanding of this threshold requires also the integration with previous knowledge on food science and food sensory together with economic implications of the choices made.

As seen from the previous analysis of the module, the threshold concepts will bring together different aspects of the subject that previously did not appear to be related (Biggs, 2003) covering the new concepts of packaging and materials, but also previous knowledge of food chemistry, technology, sensory, consumer perception and basic economics. Thus, the comprehension of these threshold concepts may transform the internal view of the content of the module by the learner and influence their future professional practice.

\section{CONSTRUCTIVE ALIGNMENT}

The variety and complexity of the thresholds identified and the need to integrate the contents of the module, while maintaining the curriculum coherence and aligning the learning activities, assessment and learning outcomes will be key for the success of the module online. The principles of constructive alignment were applied when re-designing the learning activities and assessment strategy of this module to an online environment. Constructive alignment has been defined as an outcome-based approach in which the learning outcomes are defined before the teaching takes place and the teaching activities and assessments are designed 
to guide the learners to achieve them (Biggs, 2014). This theory is based on the principles of alignment between course objectives and assessment targets defined by instructional designers (Biggs, 1996) and previous teaching philosophies of constructivism. Constructivism focuses on developing teaching activities that create meaning or learning by doing defined by Tyler (2013) as "learning takes place through the active behavior of the learner: it is what he does that he learns, not what the teacher does". The alignment of the learning outcomes, learning activities, assessment and grading of the re-designed module online are summarized in Figure 1.

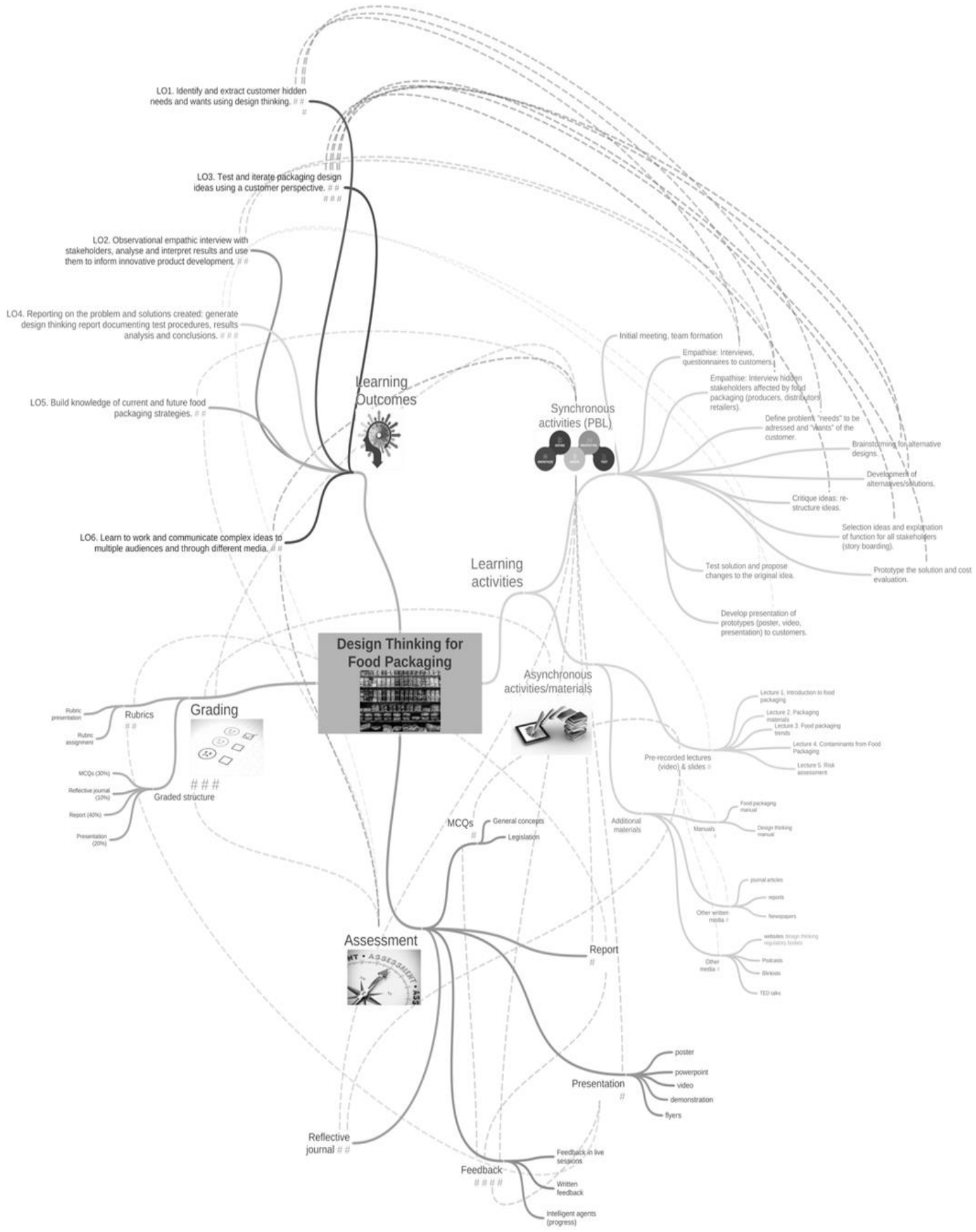

Figure 1. Mind map analyzing the relationships between multiple elements of the re-designed module online 


\section{IMPLEMENTATION PLAN}

\subsection{Online Learning Activities}

\subsubsection{Synchronous Learning Activities}

The learners were divided into groups of 6-5 to work together as part of a "food consultant group" for a period of 10 weeks. The groups of learners were then adhered to 2 cohorts that were posed with 2 different problem based learning (PBL) scenarios related to the packaging of different food products. Moreover, the learners were also introduced to the methodology of design thinking, designed by Hasso Plattner Institute of Design at Stanford University (https://dschool.stanford.edu/), to solve the PBL. Design thinking is a methodology for creative problem solving aiming to maximize creativity and innovation in industry settings counteracting human biases that hinder creativity through strong emphasis on empathy with clients, end users and innovators, while addressing the challenges of costs-risks and employee buy-in (Liedtka, 2018). This methodology comprises five steps - empathize, define, ideate, prototype, test and assess (Hasso Plattner, Institute of Design at Standford) - and include specific activities within each step that will act as the main scaffoldings during the PBL process as represented in Figure 2.

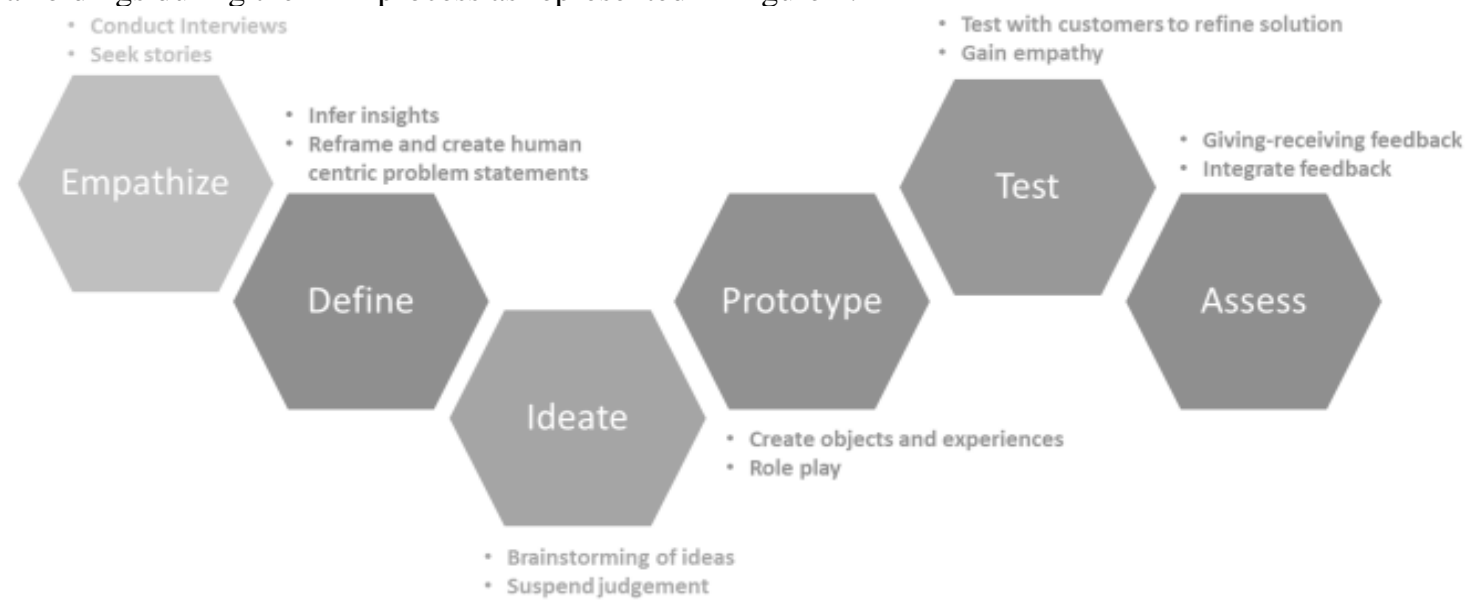

Figure 2. Design thinking methodology steps followed for problem solving. The content of this figure was modified from the Institute of design of Stanford University

(https://engineering.stanford.edu/get-involved/give/hasso-plattner-institute-design, https://dschool.stanford.edu/)

The PBL process was guided through online sessions (synchronous learning) via Zoom and the group activities of the learners were facilitated via break-out rooms available in this online platform. The online activities facilitated the discussion and brainstorming of the groups and allowed the module coordinator to provide feedback on each group's performance towards achieving the main learning outcomes of each session. Moreover, the design thinking approach includes additional activities ("empathic interviews", questionnaires and formal interviews to stakeholders related to food packaging) involving "action learning", as the learners will have to engage with external stakeholders and gain insights to understand the problem further and propose better solutions and prototypes. This "action learning" required the development and practice of communication and interpersonal skills and an increased empathy with stakeholders affected by the food package (consumers, distributors, retailers and waste collectors). All these learning activities helped the learners to achieve a deeper understanding of the problem posed and to apply their knowledge into key thresholds identified in the discipline - food processing and manufacturing; consumer acceptability and market - while contributing also to identify areas for further research during their PBL - food products and raw materials, food packaging materials and regulations - in order to successfully design their packaging and solve the problems posed.

\subsubsection{Asynchronous Learning Activities}

The online synchronous learning activities were complemented with 6-8 hours of pre-recorded lectures (short videos, full lectures, slides and notes) and other additional learning resources (reading materials, videos, 
blogs, podcasts and web-sites/online publications of professional associations and regulatory bodies related to food packaging) shared with the learners during 10 weeks via the online platform of the module (Brightspace). The lectures and additional resources served as a theoretical guidance on new concepts related to the threshold concepts previously identified (1) food products and raw materials, (2) food packaging materials and regulations, (3) food processing and manufacturing and (4) consumer acceptability and market. These asynchronous learning activities and resources were relevant for the learners as they had to use them to advance in their packaging knowledge to develop potential solutions to the problems covered during the synchronous sessions. Thus, the asynchronous learning served as stepping stone towards a deeper understanding of key concepts that the learners will have to tailor and apply specifically to their PBL, researching further and exploring in depth their specific food products with different packaging needs during the synchronous sessions.

\subsection{Assessment Strategy}

The assessment strategy of the re-designed "Design Thinking for Food Packaging" online reflected both the individual and collective efforts towards achieving the learning outcomes of the module. Thus, the assessment strategy was aligned with the learning activities and learning objectives of the module and it was implemented as:

(1) Individual assessment: multiple choice questions (MCQs) and online learning journal. The new theoretical content of the module delivered through the asynchronous learning activities was evaluated by MCQs performed via the online platform of the module (Brightspace). This allowed an individual assessment of the progress of each learner on the theoretical contents of the module. Moreover, as part of their individual assessment, the learners were introduced to the "reflective practice" via an electronic learning journal. This journal included nine entries in which each learner reflected on their own individual learning journey by identifying key experiences, learning acquired as result of those experiences and how this learning will be applied in their future professional practice. This assignment also allowed an individual assessment of the learners' achievements in relation to their interpersonal skills as they reflected on different aspects of group work (i.e. problem solving, communication, time management, organization) and engagement with stakeholders through the proposed "action learning" activities (i.e. interviews to stakeholders with different cultural backgrounds and professional relationships established during this process).

(2) Group assessment: presentation session and portfolio of group work. The assessment of the group work was done in a presentation session (poster, video, PowerPoint) in which the learners presented to an audience the packaging prototype developed by the group as well as the details on how the packages work and the specific problem/s that the newly developed prototype solves. This presentation session was performed online via Zoom with invited speakers from multiple industries that posed questions to the groups on their work. Moreover, the learners also generated a full portfolio of work based on their project using several collaborative platforms (Dropbox or shared drive in Google). This portfolio followed the structure and steps of the PBL of each group and the additional research conducted in different aspects of their food packaging (i.e. regulatory/scientific aspects of packaging) that concluded with the design and presentation of the proposed prototype presented in the online session. This allowed the assessment of the application and further research performed by the learners, as well as their capacity to integrate the knowledge of all the key threshold concepts identified in the discipline to solve each of the problem based scenarios posed to the groups.

\section{CONCLUSION}

Future work is necessary to evaluate if the changes implemented in the module delivered online led to noticeable improvements in the learning experience and achievement of the learning outcomes proposed. The main tool to allow an objective evaluation of the module will be based on collecting feedback and pose specific questions related to the learning activities and assessment strategy, as well as perceived achievement of the learning outcomes, via an online questionnaire that could be compared with previous general feedback of the module evaluated by cohorts of learners evaluating the original module delivered face-to-face. 
Overall, the re-structured module online clearly aligned the existing learning objectives with new learning activities and assessment strategy online, facilitating the integration of knowledge. The new synchronous learning activities online (solving PBL by the "design thinking" methodology) were strategically designed to facilitate the learners' further understanding of the asynchronous materials (lectures and readings) as well as to engage the learners into further research involving the main threshold concepts identified within this module - (1) Food products and raw materials; (2) Food packaging materials and regulations; (3) Food processing and manufacturing; (4) Consumer acceptability and market - enabling them to establish connections between multiple disciplines of the Food Science Programme and to apply this knowledge during problem solving activities, similarly to real scenarios and problems they will face in their future professional practice.

The interpersonal skills (communication, empathy and leadership skills) of the learners were further develop through "active learning" while engaging multiple stakeholders in relation to their future packaging solutions as part of the design thinking methodology used as scaffolding for solving the PBL. These valuable learning experiences within the re-designed "Design Thinking for Food Packaging" module will feed-forward into the professional and personal development of the learners at the beginning of their careers as food scientists working in a wide variety of industries, including food companies (i.e. food processors, quality and safety control, sensory experts) and other related businesses (i.e. regulatory agencies, food safety inspectors), in which the learners will have to engage with a wide range and variety of stakeholders in their professional capacities.

\section{ACKNOWLEDGEMENT}

Marco Garcia-Vaquero would like to thank América Martín Mezquita and Blanca Vaquero Martín for their constant support, wisdom and passion for education that was passed on to everyone around them. América Martín Mezquita "Study, learn and get educated! Money can come and go; Knowledge will always stay with you and it will help you forever".

\section{REFERENCES}

Arvanitoyannis, I. S. and Kotsanopoulos, K. V., 2014. Migration phenomenon in food packaging. Food-package interactions, mechanisms, types of migrants, testing and relative legislation-a review. Food and Bioprocess Technology, 7, 21-36.

Biggs, J., 1996. Enhancing teaching through constructive alignment. Higher Education, 32, 347-364.

Biggs, J., 2003. Teaching for Quality Learning at University. SRHE and Open University Press, Buckingham, United Kingdom.

Biggs, J., 2014. Constructive alignment in university teaching. HERDSA Review of Higher Education Vol. 1, pp. 5-2.

Hasso Plattner. An Introduction to Design Thinking. Facilitator's Guide. Institute of Design at Standford, USA.

https://dschool.stanford.edu/ (Accessed $19^{\text {th }}$ November 2020).

https://engineering.stanford.edu/get-involved/give/hasso-plattner-institute-design (Accessed 19 ${ }^{\text {th }}$ November 2020).

Liedtka, J., 2018. Why Design Thinking Works. Harvard Business Review, September-October 2018 issue, pp. 72-79.

Meyer, J. and Land, R., 2003. Threshold Concepts and Troublesome Knowledge 1 -Linkages to Ways of Thinking and Practising' in Improving Student Learning - Ten Years On. C.Rust (Ed), OCSLD, Oxford, United Kingdom.

Sandhya, 2010. Modified atmosphere packaging of fresh produce: Current status and future needs. LWT - Food Science and Technology, 43, 381-392.

Sarker, D. K., 2020. Packaging Technology and Engineering: Pharmaceutical, Medical and Food Applications, John Wiley \& Sons.

Sjöö, M., Rayner, M. and Ostman, E., 2010. Concept Circuit Training: A new pedagogic approach for better teaching and learning.

Tyler, R. W., 2013. Basic principles of curriculum and instruction, University of Chicago press.

Vera, P., Canellas, E. and Nerín, C., 2020. Compounds responsible for off-odors in several samples composed by polypropylene, polyethylene, paper and cardboard used as food packaging materials. Food Chemistry, 309, 125792. 